\title{
The risks associated with erector spinae plane block in patients with abnormalities of coagulation
}

\author{
Abhijit S. Nair and Suresh Seelam \\ Department of Anesthesiology, Basavatarakam Indo-American Cancer Hospital and Research Institute, Hyderabad, \\ Telangana, India
}

We read with interest the case report titled "Erector spinae plane block combined with a novel technique for selective brachial plexus block in breast cancer surgery" by De Cassai et al. [1]. The authors described erector spinae plane block (ESPB) and selective brachial plexus (SBP) block in a 77-year-old male patient on dual antiplatelet therapy (DAPT). However, the idea of performing ESPB on a patient on DAPT is perceived as challenging. Moreover, they have performed a SBP block in which the thoracodorsal nerve (TDN), long thoracic nerve (LTN), and the medial and lateral pectoral nerves were blocked, as well. This letter describes an opinion with critical comments, citing relevant references, as well as a word of caution.

Cutaneous innervation of the breast is indeed very complex [2]. The authors have described the intercostal nerves, pectoral nerves (medial and lateral), LTN, and TDN, which are the nerves innervating the anterior chest wall. The pectoral nerves, LTN, and TDN are essentially motor nerves and need not be blocked separately for a mastectomy. Although there are no clinical issues with blocking the pectoral nerves, there are no advantages either.

The LTN is the motor nerve to the serratus anterior muscle, and an undesirable sequela of its block is winging of the

Corresponding author: Abhijit S. Nair, M.D.

Department of Anesthesiology, Basavatarakam Indo-American Cancer Hospital and Research Institute, Road No. 10 Banjara Hills, Hyderabad, Telangana 500034, India

Tel: 91-040-23351235, Fax: 91-040-23542120

Email: abhijitnair95@outlook.com

ORCID: https://orcid.org/0000-0003-2506-0301

Received: December 30, 2018.

Revised: January 7, 2019.

Accepted: January 8, 2019.

Korean J Anesthesiol 2019 June 72(3): 275-276

https://doi.org/10.4097/kja.d.18.00371 scapula, which is a possibility with an isolated LTN block. The undesirable effect of this isolated block could add to significant morbidity in the postoperative period and can affect physiotherapy as well. Similarly, the TDN is a motor nerve supplying the latissimus dorsi muscle, and its selective block has no additional role in providing analgesia. Further, its role in nociception has not been established. The authors have pointed out that an ESPB could, sometimes, spare the anterior branches of the intercostal nerves. However, that depends on the level at which the block is performed. This sparing can be addressed by using a high-volume of local anesthetic for the block (up to $30 \mathrm{ml}$ ) [3].

For patients undergoing perineuraxial, deep plexus, or deep peripheral block, the American Society of Regional Anesthesia (ASRA) guidelines have recommended the same guidelines that are applicable for neuraxial techniques [4]. The recent guidelines recommend stopping clopidogrel at least 5 days prior to neuraxial blockage. There is an increased risk of bleeding if the patient is on DAPT, i.e., aspirin with a thienopyridine. Although platelet aggregation assays are available to determine if there is any residual effect of clopidogrel even after stopping its intake, the current evidence is not sufficient to predict outcomes based on such assays.

Cadaveric and contrast studies have shown that the injectate after an ESPB eventually traverses through the fascial plane to the paravertebral space [5] (Fig. 1). The ESPB is classified under the category of a perineuraxial block as the local anesthetic (LA) eventually spreads to the epidural and paravertebral spaces and, also, shows interforaminal spread. At the thoracic level, the ESPB is performed after piercing the skin, subcutaneous tissue, trapezius, and the rhomboideus major and erector spinae muscles. Therefore, ESPB can also be classified as a deep peripheral nerve block. Therefore, we feel that performing an ESPB on a patient who has not stopped taking clopidogrel and ecosprin for at least 5 days as per ASRA guidelines is not a wise approach. A hematoma in the erector spinae plane has not been described

(c) This is an open-access article distributed under the terms of the Creative Commons Attribution Non-Commercial License (http://creativecommons.org/ licenses/by-nc/4.0/), which permits unrestricted non-commercial use, distribution, and reproduction in any medium, provided the original work is properly cited. 


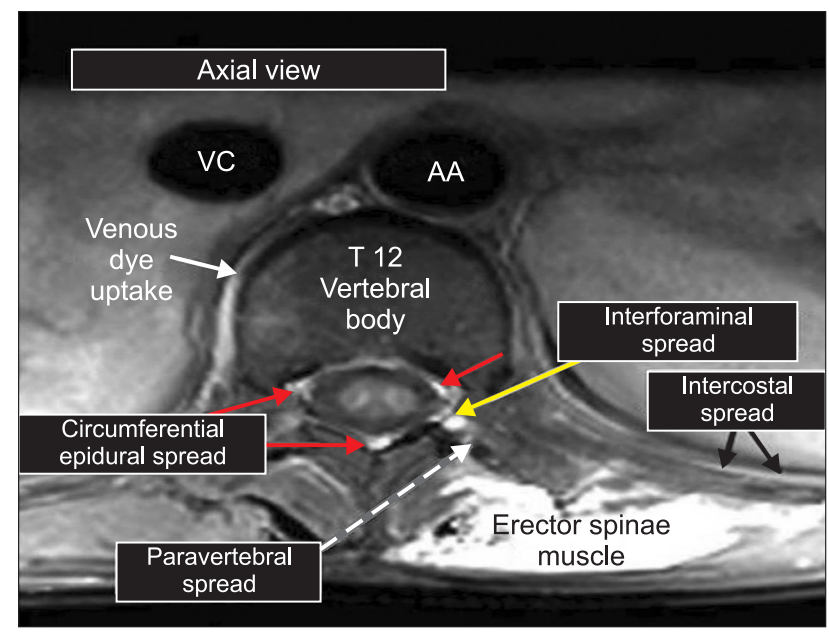

Fig. 1. Magnetic resonance imaging with gadolinium contrast showing spread of contrast to the neuraxis (epidural, paravertebral) and interforaminal spread. Therefore, an erector spinae plane block is a perineuraxial block. As ESPB is performed after traversing the skin, subcutaneous tissue, and the trapezius, rhomboideus major, and erector spinae muscles, it can be considered as a deep peripheral nerve block as well. VC: vena cava, AA: abdominal aorta. (Figure obtained with permission from 'Springer Nature', License Number: 4498681486818. Image source: "Schwartzmann et al. Mechanism of the erector spinae plane block: insights from a magnetic resonance imaging study. Can J Anaesth 2018; 65: 1165-6"). yet, but it may have disastrous consequences. Vessel injury or an underlying altered coagulation status can lead to formation of a hematoma anywhere in the course of contrast spread as shown on the MRI scan. ESPB is a recently described block, and it is rapidly being explored for novel indications. Therefore, caution is required when performing this block on coagulopathic patients and in patients taking antiplatelet or antithrombotic agents.

\section{Conflicts of Interest}

No potential conflict of interest relevant to this article was reported.

\section{Author Contributions}

Abhijit S. Nair (Conceptualization; Investigation; Supervision; Writing - original draft)

Suresh Seelam (Writing - review \& editing)

\section{ORCID}

Abhijit S. Nair, https://orcid.org/0000-0003-2506-0301

Suresh Seelam, https://orcid.org/0000-0002-0387-1464

\section{References}

1. De Cassai A, Bonvicini D, Ruol M, Correale C, Furnari M. Erector spinae plane block combined with a novel technique for selective brachial plexus block in breast cancer surgery. Korean J Anesthesiol 2019; 72: 270-4.

2. Nair AS. Cutaneous innervations encountered during mastectomy: a perplexing circuitry. Indian J Anaesth 2017; 61: 1026-7.

3. Nair AS, Seelam S, Naik V, Rayani BK. Opioid-free mastectomy in combination with ultrasound-guided erector spinae block: a series of five cases. Indian J Anaesth 2018; 62: 632-4.

4. Horlocker TT, Vandermeuelen E, Kopp SL, Gogarten W, Leffert LR, Benzon HT. Regional anesthesia in the patient receiving antithrombotic or thrombolytic therapy: American Society of Regional Anesthesia and Pain Medicine evidence-based guidelines (fourth edition). Reg Anesth Pain Med 2018; 43: 263-309.

5. Schwartzmann A, Peng P, Maciel MA, Forero M. Mechanism of the erector spinae plane block: insights from a magnetic resonance imaging study. Can J Anaesth 2018; 65: 1165-6. 